\title{
Monte Carlo Calculations of Amplification Spectrum for GaN THz Transit-time Resonance Maser
}

\author{
E. STARIKOV ${ }^{\mathrm{a}, \mathrm{c}}$, P. SHIKTOROV ${ }^{\mathrm{a}}$, V. GRUŽINSKIS ${ }^{\mathrm{a}}$, L. REGGIANI $^{\mathrm{b}, *}$, L. VARANI $^{\mathrm{c}}$, \\ J. C. VAISSIÈRE ${ }^{\mathrm{c}}$ and JIAN H. ZHAO ${ }^{\mathrm{d}}$ \\ ${ }^{a}$ Semiconductor Physics Institute, A. Goštauto 11, 2600 Vilnius, Lithuania; \\ ${ }^{\mathrm{b}}$ Dipartimento di Ingegneria dell'Innovazione, Istituto Nazionale di Fisica della Materia, Università \\ di Lecce, Via Arnesano s/n, 73100 Lecce, Italy; ${ }^{\circ}$ Centre d'Electronique et de Micro-optoélectronique \\ de Montpellier, (CNRS UMR 5507) Université Montpellier II, 34095 Montpellier \\ Cedex 5, France; ${ }^{\mathrm{d}}$ SiCLAB, Department of Electrical and Computer Engineering, \\ Rutgers University, Piscataway, NJ 08855, USA
}

\begin{abstract}
We report Monte Carlo calculations of the amplification spectrum of microwave generation in bulk $\mathrm{GaN}$ and its dependence on applied electric fields, doping level, lattice temperature, etc. The amplification is shown to occur in a wide frequency range of 0.05 to $3 \mathrm{THz}$ with an optimal generation efficiency of about $1 \sim 2 \%$.
\end{abstract}

Keywords: THz generation; GaN; Monte Carlo simulation

\section{INTRODUCTION}

During the last decades significant efforts have been devoted to realize a tunable $\mathrm{THz}$ semiconductor radiation source in view of its broad range of applications. In bulk semiconductors, a physical mechanism appropriate to this purpose is the so called optical phonon transit-time resonance (OPTTR) [1]. It consists of the periodic motion of carriers inside the optical phonon sphere of momentum space, resulting from the combined action of carrier quasi-ballistic acceleration by an applied electric field up to the optical phonon energy and the subsequent emission of an optical phonon, which pushes the carrier back near the sphere center. As a consequence, the motion takes an oscillatory character and a dynamic negative differential mobility (DNDM) can appear at frequencies near the inverse of the transit-time and its harmonics [1]. In standard III-V semiconductors, such as GaAs and InP, the maximum generation frequency was found to be limited in the range 300 to $400 \mathrm{GHz}$ [2]. By contrast, for the wide-gap materials, such as $\mathrm{GaN}, \mathrm{InN}, \mathrm{SiC}$, etc., one can expect a considerable increase of the maximum generation frequency and a general improvement of the conditions for the DNDM to occur, as a result of a higher value of the optical

*Corresponding author. Tel.: (3702) 614920, Fax: (3702) 627123, e-mail: jane@pav.pfi.lt 
phonon energy and a stronger interaction of electrons with optical phonons. The aim of this work is to confirm the above expectation by calculating the amplification band and the maximum gain for an OPTTR maser based on bulk zincblende and wurtzite GaN.

\section{COMPUTATIONAL PROCEDURES}

In general, the amplification spectrum (or the gain, $\alpha(f)$ ) of microwaves (MW) which can propagate in some active medium is described by the frequency dependence of the real part of the carrier MW mobility, $\operatorname{Re}[\mu(f)]$, as:

$$
\alpha(f)=-\operatorname{Re}[\mu(f)] n \frac{e}{c \varepsilon_{0} \sqrt{\varepsilon}}
$$

where $n$ is the carrier concentration, $c$ the light velocity in vacum, $\varepsilon_{0}$ the permittivity of vacuum, and $\varepsilon$ the static dielectric constant of the material. Under linear conditions, $\alpha(f)$ determines the frequency region of amplification, gives a threshold value of the net losses for generation to appear, allows one to choose the optimal doping level $N_{D}$ of a sample, etc. Under nonlinear conditions, when $\alpha(f)$ depends on the MW field amplitude, it allows one to determine the energy and power characteristics of generation. Under multi-signal operation it allows one to determine the characteristics of each radiation mode, the spectral behavior of the amplification band under single-mode generation, etc.

\section{Small-signal Response}

Under linear conditions the MW mobility is independent of the MW field amplitude and is determined by the Fourier transform of the linear response function $K_{x x}(s)$ as [2,3]:

$$
\mu_{x x}(\omega)=e \int_{0}^{\infty} K_{x x}(s) \exp (-i \omega s) d s
$$

where $\omega=2 \pi f$ is the circular frequency, and only the longitudinal velocity response in the direction of the constant applied electric field $\mathbf{E}_{0}=\left(E_{0}, 0,0\right)$ is taken to be of interest. In turn, when the singleparticle history is simulated by the Monte Carlo (MC) method, the longitudinal linear response function can be expressed in terms of velocity averaging over before- and after-scattering ensembles as [2]:

$$
K_{x x}(s)=\frac{1}{e E_{0}\langle\tau\rangle}\left[\left\langle v_{x}(s)\right\rangle_{b}-\left\langle v_{x}(s)\right\rangle_{a}\right]
$$

where

$$
\begin{gathered}
\left\langle v_{x}(s)\right\rangle_{b}=\frac{\langle\tau\rangle}{T-s} \int_{s}^{T} \nu[\mathbf{p}(t-s)] v_{x}[\mathbf{p}(t)] d t \\
\left\langle v_{x}(s)\right\rangle_{a}=\frac{1}{N} \sum_{i=1}^{N} v_{x}\left(t_{i}+s\right)
\end{gathered}
$$

are the probable velocities of a carrier at time $s$ under the condition that at time $s=0$ it was just before or just after a scattering event, respectively. Here $N$ is the total number of scattering events in the time interval $[0, T]$ simulated by the MC procedure, $\langle\tau\rangle=T / N$ the mean time of free flight, $\nu(\mathbf{p})$ the scattering rate for momentum $\mathbf{p}, t_{i}$ the time moments of scattering events. Then, substitution of $\operatorname{Re}[\mu]$ into Eq. (1) gives the static gain, $\alpha_{0}(f)$, defined in the absence of the MW field. The advantage of this procedure is that, for a given $E_{0}$ and $N_{D}$, it allows one to obtain the whole amplification/absorption spectrum during a single MC simulation.

\section{Large-signal Response}

Under nonlinear conditions, the carrier mobility depends on the MW field amplitude and the MW mobility is directly calculated from the velocity response. For this sake the MW electric field of the amplified mode $E_{m w}(t)=\operatorname{Re}\left[E_{\omega} \exp (i \omega t)\right]$ is directly introduced into the equation of motion $\dot{p}_{x}(t)=e E_{0}+e E_{\omega} \cos (\omega t)$, which describes the free 
flight of a trial particle in momentum space. During the simulation of a single-particle random trajectory $\mathbf{p}_{t}$ the sampling time of the average drift velocity is determined by averaging over a large number $M$ of MW electric field periods with duration $T_{\omega}$

$$
\langle v(s)\rangle_{\omega}=\frac{1}{M} \sum_{i=0}^{M-1} v_{x}\left(\mathbf{p}_{t=s+T_{\omega} i}\right)
$$

where $s$ belongs to the time interval $0 \leq s<T_{\omega}$. In accordance with the Fourier analysis, the real part of the velocity response is calculated as:

$$
v_{\omega}^{r}=\frac{2}{T_{\omega}} \int_{0}^{T_{\omega}}\langle v(s)\rangle_{\omega} \cos (\omega s) d s
$$

In this case the MW mobility is determined from the real part of the velocity response as: $\operatorname{Re}\left[\mu_{\omega}\right]\left(E_{\omega}\right)=v_{\omega}^{r} / E_{\omega}$. The dynamical gain, $\alpha_{d}\left(E_{\omega}\right)$, which already depends on the MW field amplitude, is then calculated from $\operatorname{Re}\left[\mu_{\omega}\right]$ by using Eq. (1).

Another relevant quantity, which can be calculated under large-signal operation at a given frequency, is the power generated by an active medium, $P_{\omega}\left(E_{\omega}\right)=e n v_{\omega}^{r} E_{\omega} / 2$, which is negative for generation. To analyze the stable generation of an active medium placed inside an external resonant system, it is often suitable to eliminate $E_{\omega}$ from $P_{\omega}\left(E_{\omega}\right)$ and $\alpha_{d}\left(E_{\omega}\right)$, i.e., to express $P_{\omega}$ as a function of $\alpha_{d}$ only. Under stable generation, the dynamic gain is equal to the coefficient of the net loss in the resonant system, $\alpha_{L}=\Gamma+\Lambda$. It includes both the outside power extraction, as described by coefficient coupling the active medium with the external free space, $\Gamma$, and all the parasitic losses, as described by the coefficient of internal losses, $\Lambda$. Therefore, one can finally represent the power generated inside the resonator as a function of $\alpha_{\mathbf{L}}$ by: $P_{\text {gen }}\left(\alpha_{L}\right)=-P_{\omega}\left(\alpha_{d}\right)$. In such case, the outside power extraction is given by:

$$
P_{\text {out }}=P_{\text {gen }}\left(\alpha_{L}\right) \frac{\Gamma}{\Gamma+\Lambda}
$$

The generation efficiency, $\eta$, is estimated as $\eta=P_{\text {gen }} / P_{0}$, where $P_{0}=e n\langle v\rangle_{0} E_{0}$ is the total constant power absorbed by the sample, and $\langle v\rangle_{0}$ the average velocity of carriers.

\section{Multi-signal Response}

To analyze the interaction among various possible modes in a resonant system it is necessary to investigate the system response in the presence of two or more strong signals. Accordingly, to find the conditions favourable for a single-mode operation of a maser, it is often preferable to know the behavior of the small-signal amplification spectrum under the stable single-mode generation operation, i.e., in the presence of a large-amplitude MW electric field at a given frequency. In such case, MC simulations are carried out by applying several MW fields at different frequencies $\omega_{j}$, i.e., by taking the electric field in the form of $E(t)=E_{0}+\sum_{j} E_{\omega_{j}} \cos \left(\omega_{j} t+\varphi_{j}\right)$. Then, similarly to the previous case, independent velocity averagings in accordance with Eq. (6) are performed in parallel to each frequency, so that further calculations of the MW mobility and generated power for the given frequency set can be performed separately. For example, by applying one strong signal at frequency $\omega$ and scanning the amplification spectrum with another small signal at different frequencies $\omega^{\prime}$, one can study the variations of the amplification spectrum under stable generation.

\section{NUMERICAL RESULTS}

The above computational procedures are applied to calculate the amplification and generation spectra in bulk zincblende and wurtzite $\mathrm{GaN}$ at low lattice temperatures under OPTTR conditions. The parameters of the band models are taken from Refs. [4, 5]. For acoustic and polar optical phonon scatterings the usual expressions are used [6]. The scattering mechanism from ionized impurities has been numerically optimized to shorten the cpu 
time associated with the large number of small angle scatterings. Moreover, due to the essential role played by impurity scattering, three models of screening have been checked, namely: ConwellWeiskopf, Brooks-Herring with the lattice temperature and Brooks-Herring with the electron temperature determined by the average energy of electrons. At low doping levels $\left(N_{d} \leq 10^{15} \mathrm{~cm}^{-3}\right)$ all three models give the same result in a wide range of electric fields where runway from lowenergy impurity scattering already takes place. With the increase of doping level, the most severe restrictions for the onset of OPTTR conditions are found in the Conwell-Weiskopf approximation. Therefore, below we present only numerical results obtained under this approximation, since other models of screening will lead only to improve OPTTR conditions.

\section{Linear Operation}

Calculations of the differential mobility spectrum show that the OPTTR manifests itself through a sequence of minima corresponding to the first and higher harmonics of the resonant value $\nu_{E}=1 / \tau_{E}$, where $\tau_{E}=p_{0} / e E_{0}$ is the time of an electron free flight from the center of the optical phonon sphere to its boundary. However, the broadening of the minima increases significantly for higher harmonics so that DNDM is usually achieved in the first minimum only, with the exception of the low-field region $E_{0} \leq 2 \mathrm{kV} / \mathrm{cm}$ where DNDM is found to take place also at the second harmonic. Figure 1 shows the field dependence of the maximum gain $\alpha(f)$ for the first and second amplification bands of a zincblende GaN sample with $N_{D}=10^{15} \mathrm{~cm}^{-3}$ at $T_{0}=10 \mathrm{~K}$ (curves 1 and 2 in the lower part of the figure) and the amplification range for these bands (regions bounded by curves 4, 5 and 6,7 for the first and second amplification bands, respectively, in the upper part of the figure). At low doping levels $\left(N_{D} \leq 10^{15} \mathrm{~cm}^{-3}\right)$ the gain appears at sufficiently low electric fields $E_{0} \approx 200 \mathrm{~V} / \mathrm{cm}$ and quickly reaches the maximum values at $E_{0} \approx 400 \mathrm{~V} / \mathrm{cm}$. The appearance and rapid growth

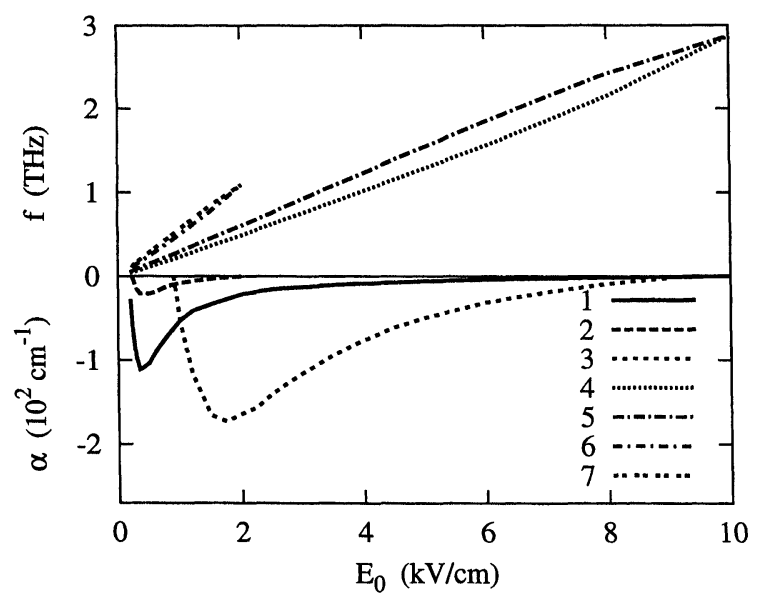

FIGURE 1 Maximum values of the gain in the first and second amplification bands (curves 1 and 2 ) as well as minimum $(4,6)$ and maximum $(5,7)$ frequencies of amplification for these bands calculated for zincblende $\mathrm{GaN}$ as a function of the electric field $E_{0}$ at $T_{0}=10 \mathrm{~K}$ and $N_{D}=10^{15} \mathrm{~cm}^{-3}$. The variation of the gain with the increase of the doping level is illustrated by curve 3 calculated for $N_{D}=10^{16} \mathrm{~cm}^{-3}$.

of the gain is connected with the onset and dominant role played by electron runaway from the low-energy region dominated by impurity scattering and the concomitant formation of a needle-like distribution in momentum space. With a further increase of $E_{0}$, the maximum value of the gain decreases approximately as $1 / E_{0}$ and finally vanishes at $E_{0} \approx 10 \mathrm{kV} / \mathrm{cm}$ due to the significant penetration of carriers into the active region, which destroys the coherence of successive free flights. Both the first and second amplification bands are very narrow (see the frequency regions bounded by curves 4,5 and 6,7 ), and the optimal amplification frequency corresponding to the maximum gain in the band increases almost linearly with $E_{0}$. As evidenced by curves 4 and 5 at low doping levels the amplification covers practically both millimeter and submillimeter ranges due to the rather wide range of electric fields corresponding to the appearance of amplification. With the increase of the doping level and, hence, of the impurity scattering efficiency, the threshold field for the onset of amplification is shifted to higher values (see curve 3 calculated for $N_{D}=10^{16} \mathrm{~cm}^{-3}$ ). After runaway from the 
low-energy region has taken place, the DNDM for low and high impurity concentrations practically coincide, since further restrictions to the occurrenc of DNDM are primarily related to carrier penetration into the active region, and these are similar in both cases. However, the gain for the high doped sample increases significantly due to the increase of carrier concentration.

From the experimental point of view, it is often easier to fix the frequency (e.g., by taking a selective detector with maximum sensitivity in a very narrow frequency range) and investigate the absorption (or amplification) of the MW radiation as function of the constant electric field applied to the sample. In this case also, the resonant character of the DNDM manifests itself as a series of resonant peaks and minima. This behavior is illustrated in Figure 2, which presents the field dependence of the amplification/absorption coefficient at a frequency of $f=1 \mathrm{THz}$ calculated by the first MC procedure at $T_{0}=10 \mathrm{~K}$ for wurtzite $\mathrm{GaN}$ with $N_{D}=10^{16} \mathrm{~cm}^{-3}$ (curve 1). Again, amplification is achieved in a narrow range of electric field values. We check the accuracy of calculations by

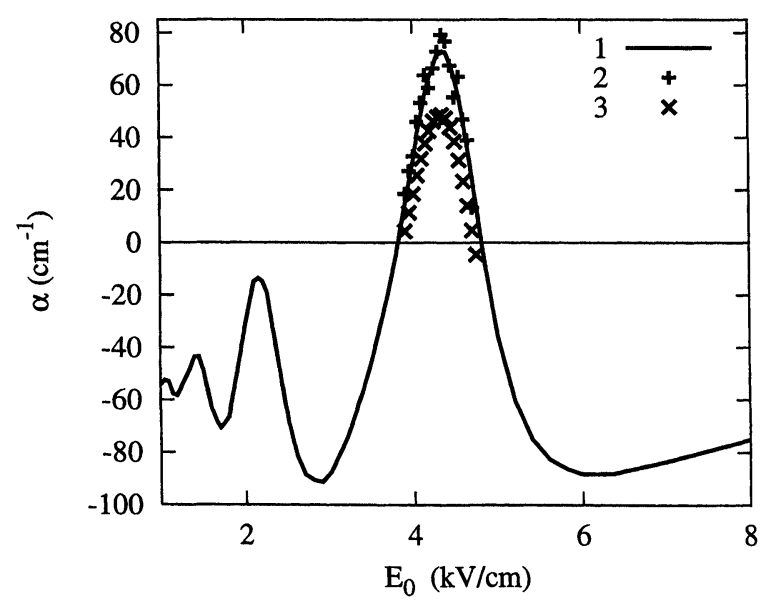

FIGURE 2 Amplification (positive) and absorption (negative) coefficients at $1 \mathrm{THz}$ frequency as a function of the static electric field strength for wurtzite $\mathrm{GaN}$ calculated by using: (i) the averaging over before- and after-scattering ensembles (curve 1), and (ii) the velocity response to a MW electric field with an amplitude of 0.2 and $3 \mathrm{kV} / \mathrm{cm}$ (curves 2 and 3 , respectively) at $T_{0}=10 \mathrm{~K}$ and $N_{D}=10^{16} \mathrm{~cm}^{-3}$. using various procedures, and to illustrate the change in amplification at large MW field amplitudes curves 2 and 3 show $\operatorname{Re}[\mu]$ calculated directly from the velocity response to a harmonic MW field with amplitudes $E_{\omega}=0.2$ and $3 \mathrm{kV} / \mathrm{cm}$, respectively. At small amplitudes, the differential mobilities calculated by the two procedures practically coincide (curve 2). At large amplitudes the mobility decreases monotonously in the whole amplification band and the amplification region becomes more narrower (see next section for a more detailed discussion concerning nonlinear effects).

\section{Nonlinear Operation}

The investigation of the regime of stable generation is carried out by a direct simulation of the carrier response to a $\mathrm{MW}$ electric field at a given frequency as described above. Qualitatively, the general situation can be described as follows. In the presence of DNDM, an external resonant system will lead to the onset and growth of MW oscillations accompanied with an increase of the amplitude of $E_{\omega}$ inside the sample. In the initial stage, this growth will be accompanined with an increase of the MW power $P_{\omega}$ which, under small-signal conditions, is proportional to $\operatorname{Re}\left[\mu_{\omega}\right] E_{\omega}^{2}$. However, a considerable increase of $E_{\omega}$ will lead to a decrease of the dynamical gain. Finally, the full disappearance of the amplification effect will take on when both the gain and the generated power will vanish. Therefore, it becomes mandatory the choice of the resonator characteristics and of the output power generated by the sample under the constraint to provide the maximum generated power inside the sample. Figure 3 summarizes the results at the basis of such a choice by presenting the maximum generated power density, $P_{\text {gen }}$ at four differenct frequencies (and respectively, four different values of applied static fields, $E_{0}=1.2,2.25,4.3$, $6.35 \mathrm{kV} / \mathrm{cm}$ ) as a function of the coefficient of net losses in the resonant system, $\alpha_{L}$. For each curve the carrier concentration was chosen to get 


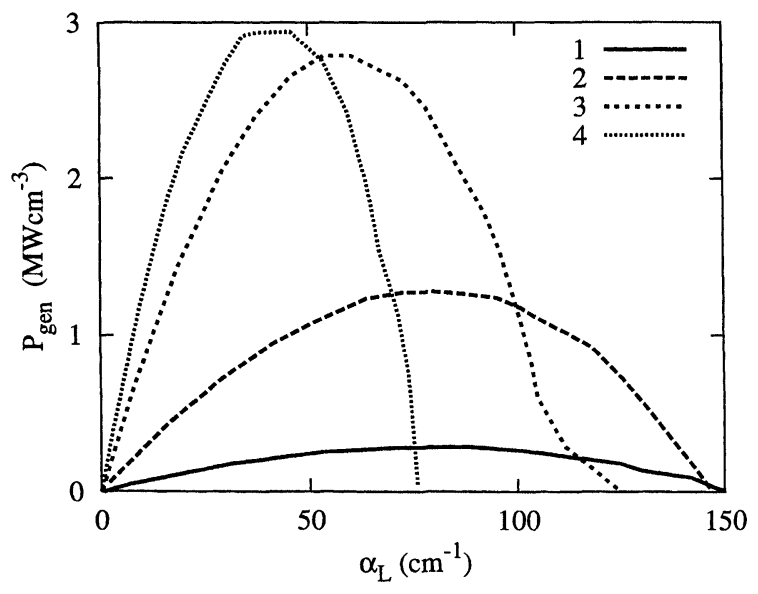

FIGURE 3 Power generated at the fundamental frequencies of $f=0.25,0.5,1$ and $1,5 \mathrm{THz}$ (cuves 1 to 4 ) inside a resonator as a function of the coefficient of total losses inside the resonator, $\alpha_{L}$, calculated in wurtzite $\mathrm{GaN}$ with the respective doping concentrations of $N_{D}=0.6,1,2$, and $3 \times 10^{16} \mathrm{~cm}^{-3}$ at $T_{0}=10 \mathrm{~K}$.

the maximum values of $P_{\text {gen }}$. Let us recall that for a stable generation the dynamical gain must exactly compensate all the losses in the resonant system due to power extraction outside the resonator, parasitic, etc., i.e., $\alpha_{d}=\alpha_{L}$. Thus, if $\alpha_{L}$ exceeds the static gain given by the linear theory then any generation is absent. Therefore, with the decrease of $\alpha_{L}$, at each given frequency generation starts from the value of the static gain. With a subsequent decrease of $\alpha_{L}$, the power increases and reaches a maximum value at the optimum amplitude of the MW field. As follows from Figure 3, this optimum value is achieved when $\alpha_{L} \simeq 0.5 \alpha_{d}$. Here, a generation efficiency of about $1 \sim 27 \%$ is achieved. With a further decrease of $\alpha_{L}$, the amplitude of the MW field increases so considerably that it would destroy the transit-time resonance. For this reason, the generated power decreases to finally vanish at $\alpha_{L}=0$.

Figure 4 compares the amplification spectra calculated when only a static field of $E_{0}=4.3 \mathrm{kV} /$ $\mathrm{cm}$ is applied (solid line), and when a strong MW field $E_{\omega}=3 \mathrm{kV} / \mathrm{cm}$ at frequency $1 \mathrm{THz}$ is superimposed on the static field (crosses). One can see that in the latter case the amplification spectrum is systematically shifted down at all frequencies,

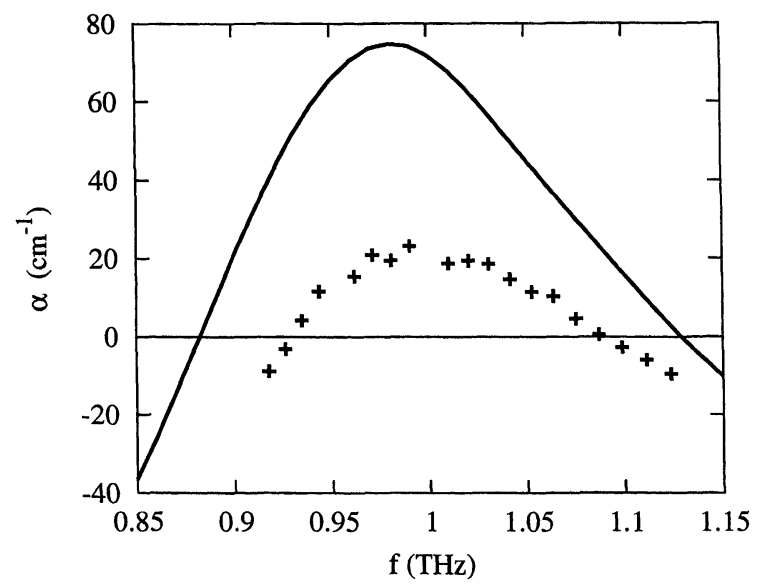

FIGURE 4 Amplification spectra under steady-state conditions at $E_{0}=4.3 \mathrm{kV} / \mathrm{cm}$ (solid line) and under a strong MW field of $E_{\omega}=3 \mathrm{kV} / \mathrm{cm}$ at $1 \mathrm{THz}$ frequency calculated for wurtzite GaN with $N_{D}=10^{16} \mathrm{~cm}^{-3}$ at $T_{0}=10 \mathrm{~K}$.

which is a useful property to obtain the singlemode generation.

\section{CONCLUSIONS}

We have presented three numerical procedures to calculate the amplification spectrum of microwave radiation under various conditions with application to an OPTTR GaN THz maser. Results confirm that $\mathrm{GaN}$ is a promising material for $\mathrm{THz}$ power generation. The MW amplification and generation caused by OPTTR occurs in the wide frequency range of 0.05 to $3 \mathrm{THz}$ and persists in the $\mathrm{THz}$ frequency range up to liquid nitrogen temperatures and doping levels of about $5 \times 10^{16} \mathrm{~cm}^{-3}$.

\section{Acknowledgments}

Authors acknowledge the support of the NATO collaborative-linkage grant PST.CLG.976340, the high-level grant DRB4/MDL/no 99-30 of the french Ministere de l'Education nationale, de la recherche et de la technologie, the french-lituanian bilateral cooperation n. 5380 of french CNRS, MADESS II project of the Italian Consiglio Nazionale delle Richerche (CNR). 


\section{References}

[1] Andronov, A. A. and Kozlov, V. A. (1973). Pis'ma ZhETF, 17, 124.

[2] Pozhela, Yu., Starikov, E. and Shiktoov, P. (1992). Sem. Sci. Tech., 7, B386.

[3] Price, P. J. (1983). J. Appl. Phys., 54, 3616.
[4] Foutz, B. E., Eastman, L. F., Bhapkar, U. V. and Shur, M. S. (1997). Appl. Phys. Lett., 70, 2849.

[5] Albrecht, J. D., Wang, R. P., Ruden, P. P., Farahmand, M. and Brennan, K. F. (1998). J. Appl. Phys., 83, 4777.

[6] Fawcett, W., Boardman, A. D. and Swain, S. (1970). J. Phys. Chem. Solids, 31, 1963. 

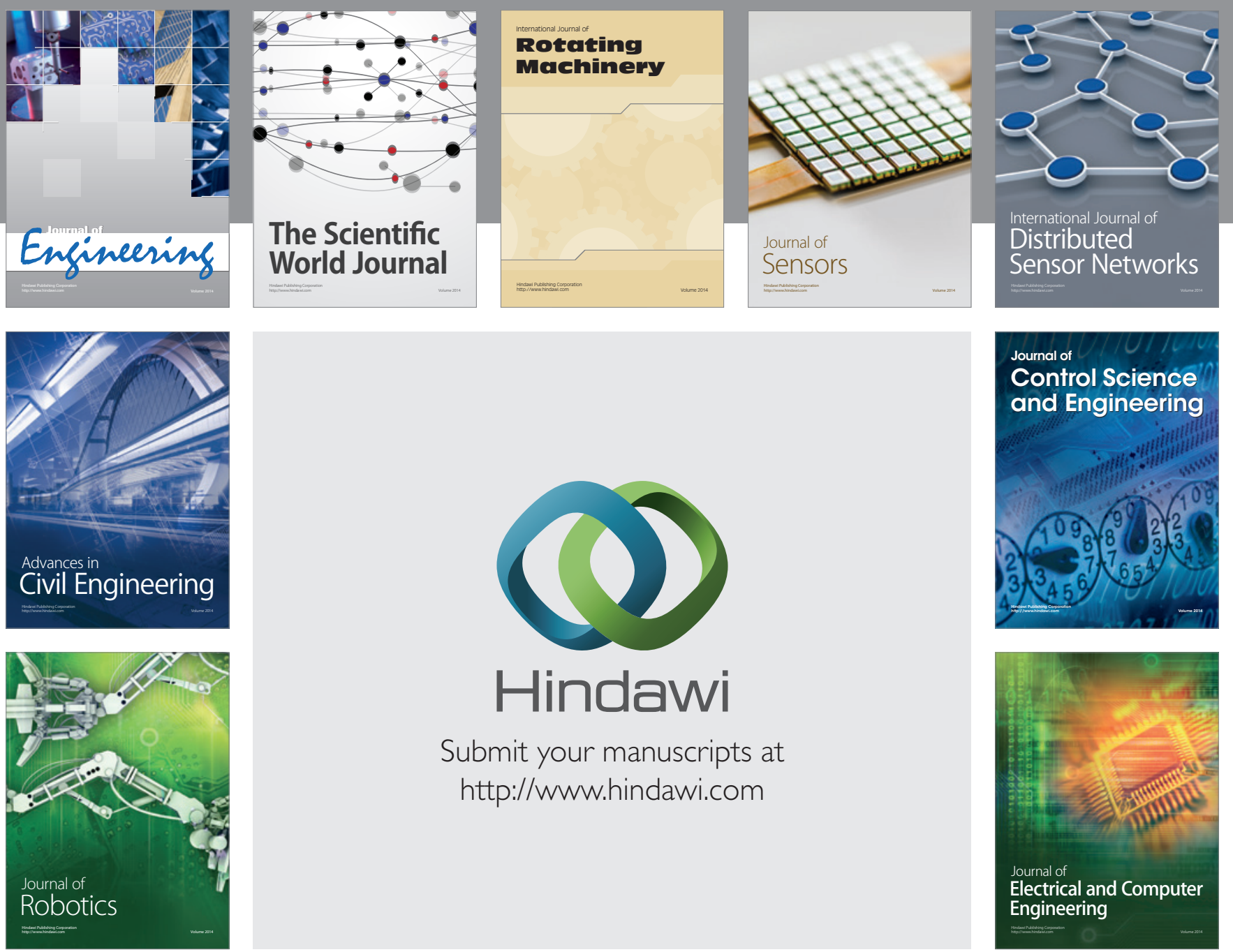

Submit your manuscripts at

http://www.hindawi.com
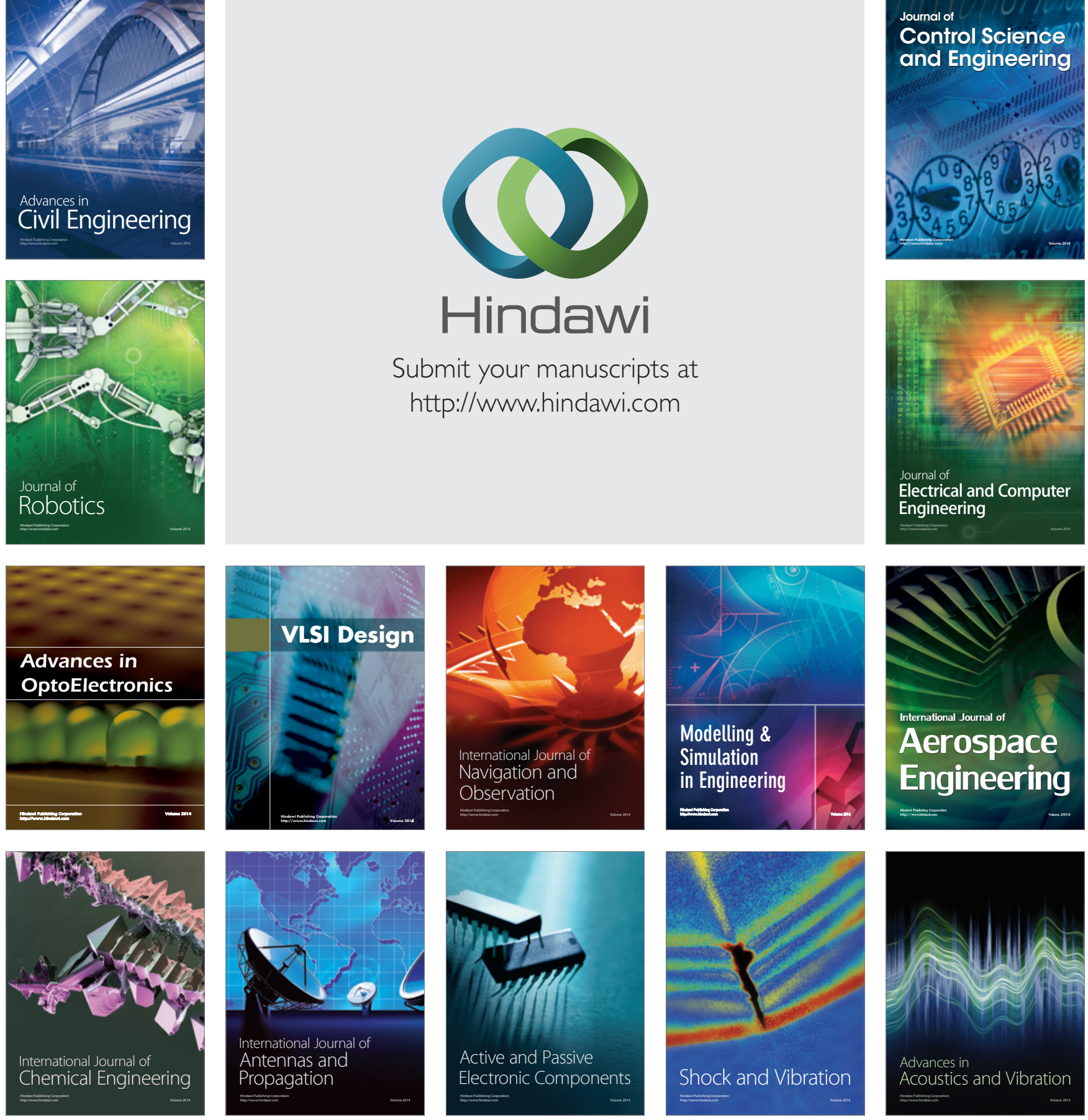